\title{
ESTUDO E MODELAGEM DO CRESCIMENTO EM DIÂMETRO DE POVOAMENTOS DE Pinus taeda L. SUBMETIDOS A PODAS E DESBASTES NA ARGENTINA
}

\author{
STUDY AND MODELING OF THE DIAMETER GROWTH IN Pinus taeda L. STANDS UNDER \\ PRUNING AND THINNING IN ARGENTINA
}

\author{
Raul Vicente Pezzutti ${ }^{1}$ Frederico Dimas Fleig ${ }^{2}$ Cesar Augusto Guimaraes Finger ${ }^{3}$ \\ Paulo Renato Schneider ${ }^{4}$
}

\begin{abstract}
RESUMO
A presente pesquisa foi realizada com o objetivo de estudar e modelar o efeito dos desbastes pré-comerciais e das podas no crescimento diamétrico, em um povoamento de Pinus taeda L. de 15 anos de idade, no Nordeste da Argentina. O estudo localizou-se em solo vermelho argiloso profundo com um delineamento sistemático de faixas para intensidades de desbastes e subfaixas para intensidades de podas. Os tratamentos de desbaste, realizados aos 3 anos de idade, foram: 1666 (sem desbaste); 833; 416 e 208 árvores ha ${ }^{-1}$. As podas foram realizadas a partir dos 3 anos e com frequência anual por 2, 3 ou 4 anos consecutivos, com remoção de 30, 50 e 70\% da copa viva, respectivamente. O modelo geral obtido por regressão linear permitiu estimar o diâmetro da árvore de área basal média (dg) em função da idade com boa precisão (desvio médio absoluto em percentagem igual a 5.71), sendo as variáveis: árvores remanescentes, copa remanescente e número de podas consideradas nos modelos estimadores dos coeficientes da função geral. Aos 15 anos de idade, os diâmetros variaram entre 25.6 e $41.5 \mathrm{~cm}$, para as densidades de 1666 e 208 árvores $\mathrm{ha}^{-1}$, respectivamente, sem poda. $\mathrm{O}$ crescimento do dg foi afetado negativamente pelas podas $\mathrm{e}$ positivamente pelos desbastes.
\end{abstract}

Palavras-chave: regimes de manejo; desrama; tratamentos silviculturais.

\begin{abstract}
The aim of this research was to study and to model the effect of pre-commercial thinning and pruning on diameter growth, on a $15^{\text {th }}$ year old stand of Pinus taeda L. in northeastern Argentina. The study was established in a deep red clay soil, with a systematic design of blocks for thinning intensity and sub-blocks for pruning intensity. The thinning treatments, performed at 3 year old were: 1666 (without thinning); 833; 416 and 208 trees $\mathrm{ha}^{-1}$. The pruning were done annually, starting at 3 year old, and applied by 2, 3 or 4 consecutive years removing 30,50 and $70 \%$ of the living crown, respectively. The general model obtained by linear regression allowed to estimate, with good precision (mean absolute deviation as a percentage equal to 5.71), the quadratic mean diameter (QMD) in function of age. The variable remnant trees, remnant crown, and number of prunings were considered to estimate the general function coefficients. At 15 year old, the QMD varied from 25.6 to $41.5 \mathrm{~cm}$ to 1666 and 208 trees ha-1, respectively, without pruning. The
\end{abstract}

1 Engenheiro Florestal, Dr., Subgerente de Silvicultura de Forestal Bosques del Plata S.A., Av. Juan Manuel Fangio, 3873, Barrio San Isidro, CEP 3300, Posadas (Misiones), Argentina. rpezzutti@cmpc.com.ar

2 Engenheiro Florestal, Dr., Professor Adjunto do Departamento de Ciências Florestais, Centro de Ciências Rurais, Universidade Federal de Santa Maria, Av. Roraima, 1000, CEP 97015-900, Santa Maria (RS), Brasil. fdfleig@smail.ufsm.br

3 Engenheiro Florestal, Dr., Professor Titular do Departamento de Ciências Florestais, Centro de Ciências Rurais, Universidade Federal de Santa Maria, Av. Roraima, 1000, CEP 97015-900, Santa Maria (RS), Brasil. finger@smail.ufsm.br

4 Engenheiro Florestal, Dr., Professor Titular do Departamento de Ciências Florestais, Centro de Ciências Rurais, Universidade Federal de Santa Maria, Av. Roraima, 1000, CEP 97105-900, Santa Maria (RS), Brasil. paulors@smail.ufsm.br

Recebido para publicação em 3/11/2012 e aceito em 10/03/2015 
QMD growth was negatively affected by pruning and positively affected by thinning.

Keywords: management schemes; pruning; silvicultural treatments.

\section{INTRODUÇÃO}

O diâmetro médio quadrático (dg) é a medida do diâmetro médio da árvore convencionalmente usado em silvicultura, em vez da média aritmética do diâmetro. As razões históricas e práticas para esta convenção foram apresentadas por Curtis e Marshall (2000) destacando o uso do diâmetro da árvore de área basal media no cálculo do volume do povoamento e sua relação com a área basal e o número de árvores.

$\mathrm{O}$ diâmetro da árvore de área basal média (dg) e o número de árvores por hectare são variáveis de entrada para os diagramas de manejo de densidade dos povoamentos (DMD). Conhecendo o dg e número de árvores/ha o Engenheiro Florestal pode usar os DMD para determinar o momento adequado para o desbaste do plantio. Por outro lado, o dg é usado para estimar a produção do povoamento e o custo de colheita da madeira. Conforme Finger (1992), o diâmetro da árvore de área basal média é muito empregado nas tabelas de produção e tarifas, é fácil de ser estimado e apresenta alta correlação com a árvore do volume médio do povoamento.

Ao existir uma clara relação entre o $\mathrm{dg}$ e o diâmetro médio aritmético (SCHNEIDER; SCHNEIDER, 2008) do povoamento, o dg pode ser usado para estimar o diâmetro médio do plantio e obter as frequências por classe diamétrica quando se conhece o desvio padrão. Estas informações são usadas para determinar o volume de cada classe diamétrica e gerar tabelas de produção. As funções que permitem estimar o dg no tempo são de utilidade para conhecer os anos necessários para atingir o diâmetro objetivo do povoamento, estimar o volume final e avaliar a rentabilidade.

No Mercosul, os plantios de Pinus taeda L. têm aumentado consideravelmente nas últimas décadas. No Sul do Brasil, Nordeste da Argentina, Uruguai e Paraguai, as áreas florestadas com Pinus sp. têm sido manejadas em geral para a produção de madeira como matéria-prima para produzir celulose, compensados e produtos de aparência ou estruturas de madeira sólida processados em serrarias. Os produtos florestais de menores diâmetros são utilizados em algumas ocasiões para gerar biocombustíveis e energia. Na Argentina, a espécie mais utilizada em plantios florestais é Pinus taeda L. Conforme Elizondo (2009), a superfície plantada com Pinus sp. na província de Corrientes, Argentina, atingiu 262.940 hectares de plantios.

Os produtores florestais, como os de outras culturas, procuram maximizar a rentabilidade. Países como Nova Zelândia e Chile são exemplos claros nos quais o manejo dos plantios tem permitido agregar valor à produção florestal. Nesse contexto, as podas e os desbastes pré-comerciais e comerciais estão sendo executados durante a rotação dos plantios de Pinus sp. no Sul do Brasil, no Nordeste argentino e em países vizinhos.

As podas e os desbastes geram, em geral, diferenças de crescimento das árvores e, consequentemente, afetam a quantidade e qualidade da madeira. Conforme a qualidade, a madeira é utilizada na geração de determinados produtos finais. Cada tora produzida normalmente é classificada pelo diâmetro na ponta fina, comprimento, forma e pelo fato de se foi ou não podada, sendo também considerada a qualidade da poda, em algumas ocasiões. Em geral, para cada categoria são definidos preços específicos.

Estudando o efeito dos desbastes em plantios de Pinus taeda de 21 anos de idade, Amateis e Burkhart (2005) concluíram que os desbastes apresentam seu maior impacto na distribuição de produtos nas classes de diâmetros maiores. Tratamentos intensos de desbastes levaram todas as árvores destinadas para produção de polpa para as classes de diâmetros maiores. Por outra parte, nas árvores de classes diamétricas intermédias, os desbastes tiveram uma pequena influência na distribuição dos produtos.

De acordo com Larson et al. (2001), o principal objetivo do desbaste é aumentar a taxa de crescimento promovendo a expansão da copa. Por outro lado, a poda de galhos vivos assegura um tamanho de nó menor e um cilindro nodoso menor, mas a poda severa pode diminuir o crescimento, aumentar a proporção de lenho tardio e a densidade da madeira na parte inferior do fuste.

Segundo Tassisa e Burkhart (1997), muitos plantios de Pinus taeda nos Estados Unidos sofrem desbastes para aumentar o crescimento em diâmetro das árvores selecionadas, procurando obter 
produtos de qualidade no menor tempo possível. Os silvicultores normalmente usam simuladores de crescimento para avaliar cenários de produção e determinar a melhor prescrição técnica, por isso, é importante ter um claro entendimento do efeito dos desbastes na produção.

Costas et al. (2006) mencionam que, na província de Misiones e no Nordeste da província de Corrientes, na Argentina, nos últimos 15 anos, têm se difundido e implementado sistemas intensivos de manejo florestal em plantios de coníferas. O controle da densidade e a realização de podas para aperfeiçoar a quantidade e a qualidade da produção florestal têm mostrado serem ferramentas eficazes para satisfazer as demandas do mercado local e internacional.

No Nordeste da província de Corrientes, muitos produtores florestais, procurando produzir madeira de qualidade, estão realizando podas e desbastes sob diferentes modalidades. Nesse âmbito, não se tem clareza do efeito da intensidade das desramas e desbastes sobre o crescimento de um povoamento do Pinus taeda e consequentemente resulta difícil prognosticar a produção futura conforme diversos regimes de manejo aplicados.

Entender e quantificar a resposta em crescimento diamétrico das árvores de Pinus taeda a tratamentos que podem reduzir a capacidade fotossintética e comprometer o crescimento por competição como são as podas ou os desbastes poderá contribuir para melhorar as tomadas de decisão pelos produtores florestais.

Assim, este trabalho foi concebido com o objetivo de estudar e modelar o crescimento em diâmetro da árvore de área basal média em plantios de Pinus taeda do nordeste Argentino em diferentes combinações de intensidades de desbastes précomerciais, intensidades de desrama e número de desramas.

\section{MATERIAL E MÉTODO}

\section{O experimento}

O experimento está localizado na província de Corrientes, Departamento de Santo Tomé, na fazenda "Pariopá", da empresa Forestal Bosques del Plata S.A. (BDP). As coordenadas geográficas do plantio em estudo são $28^{\circ} 24^{\prime} 06^{\prime \prime}$ de latitude Sul e $56^{\circ} 00^{\prime} 58^{\prime \prime}$ de longitude Oeste, com uma altitude de 108 metros sobre o nível do mar. O solo é classificado na região como profundo, argiloso vermelho, sendo o relevo suave com inclinação inferior a $15 \%$. Os solos da região são caracterizados por formações de basalto, bem drenados, denominados solos vermelhos. Sua taxonomia, segundo o sistema de classificação da Soil Taxonomy (UNITED STATES DEPARTMENT OF AGRICULTURE, 1999), corresponde a um Typic Kandihumults (Ultisoles). A série de solo na classificação Argentina é a Diaz de Vivar (ESCOBAR et al., 1996). O clima da região, segundo a classificação de Köppen, é do tipo cfa, subtropical úmido, descrito como temperado chuvoso, apresentando verões muito quentes e úmidos sem estação seca (UNIVERSDAD NACIONAL DE ENTRE RÍOS, 2010). Conforme os informes meteorológicos da empresa BDP, baseados em dados dos últimos 17 anos, a temperatura média anual é de $21^{\circ} \mathrm{C}$ e a precipitação média é de 1700 $\mathrm{mm}$ por ano.

O experimento foi conduzido num plantio comercial no ano 1993, com a espécie Pinus taeda de origem Marion, procedente do Estado da Flórida, Estados Unidos. O espaçamento inicial entre plantas foi de $3 \mathrm{~m} \times 2 \mathrm{~m}$, gerando uma densidade inicial de 1666 árvores/ha. O preparo do solo foi de uma subsolagem até $90 \mathrm{~cm}$ de profundidade; o controle de plantas daninhas na área foi realizado por dois anos após o plantio e acompanhado de controle de formigas.

A área escolhida para o estabelecimento do experimento foi selecionada pelo INTA (Instituto Nacional de Tecnologia Agropecuária) e BDP, considerando o resultado do inventário florestal realizado aos 3 anos de idade. A área de plantio selecionada para estabelecer os tratamentos de desbastes e podas foi homogênea em crescimento e sobrevivência; o talhão apresentou 1630 árvores por hectare, uma área basal média de $6,6 \mathrm{~m} / \mathrm{ha}$; um diâmetro médio de $7,2 \mathrm{~cm}$ e uma altura média de 4,8 $m$ antes da aplicação dos tratamentos.

A pesquisa foi instalada distribuindo os tratamentos sistematicamente (FASSOLA et al., 2002). As instituições responsáveis pela instalação e medição anual do experimento foram BDP e o INTA. Aos 3 anos de idade, foram estabelecidas as parcelas, com tamanho variável entre 108 e $432 \mathrm{~m}^{2}$, aumentando com a redução do número de árvores por hectare, conforme o descrito na Tabela 1.

A instalação do experimento foi realizada entre os dias 12 e 21 de agosto de 1996, sendo as podas executadas com serrote e escada. 


\section{Descrição dos tratamentos}

Os fatores principais considerados no experimento foram: Número de árvores remanescentes (AR), percentagem de copa remanescente (CR) e número de podas (NP). Os níveis do fator $\mathrm{AR}$ foram: 1666 árvores/ha (testemunha sem desbaste); 833 árvores/ha; 416 árvores/ha e 208 árvores/ha. Para o fator CR os níveis considerados foram: 100 (testemunha sem poda); 70 (poda de $30 \%$ da copa viva); 50 (poda de $50 \%$ da copa viva) e 30 (poda de $70 \%$ da copa viva). O terceiro fator foi o NP com os seguintes níveis: 1 (testemunha sem poda); 2 (duas podas de frequência anual); 3 (três podas de frequência anual) e 4 (quatro podas de frequência anual). Os tratamentos foram gerados a partir da combinação parcial dos níveis dos três fatores.

Por meio de desbastes seletivos, aos 3 anos de idade, geraram-se 4 faixas de distintas densidades: 1666, 833, 416 e 208 plantas/ha. Em cada uma, realizou-se a poda de galhos vivos, em subfaixas perpendiculares às anteriores, sendo usadas 4 intensidades de remoção de copa: 0; $30 ; 50$ e $70 \%$ do comprimento da copa viva. As subfaixas, por sua vez, foram divididas em parcelas dentro das quais foram realizadas podas por 2 , 3 ou 4 anos consecutivos. Dessa maneira, foram gerados 32 tratamentos com 5 repetições, à exceção dos tratamentos da faixa de 208 árvores/ha, que apresentaram 6 repetições. O número de plantas por parcela foi variável em função do tratamento de desbaste aplicado, oscilando entre 18 e 9 plantas. As faixas foram desenhadas com bordadura perimetral dupla entre si, e dentro de cada faixa de densidade as parcelas correspondentes a cada tratamento de poda apresentaram bordadura perimetral simples.
Na Tabela 1 estão, de forma resumida, as densidades (número de árvores por hectare), o espaço disponível por árvore individual, características das parcelas e repetições dos tratamentos correspondentes a cada faixa de densidade.

Nos casos em que o número de árvores observadas aos 3 anos de idade apresentou diferenças maiores a $17 \%$ com relação ao valor teórico do tratamento, as parcelas foram retiradas da análise. Com essa metodologia foram selecionados 30 tratamentos para serem avaliados.

A descrição dos 30 tratamentos é apresentada no capítulo de resultados e discussão (Tabela 5) sendo exibido para cada tratamento o número de árvores remanescentes após o desbaste (AR); a percentagem de copa viva remanescente após a realização da poda $(\mathrm{CR})$ e o número de podas (NP) executado segundo a seguinte descrição: 1 (sem poda, correspondente à testemunha); 2 (duas podas de frequência anual); 3 ( 3 podas de frequência anual) e 4 (máximo nível de poda, sendo realizadas 4 podas de frequência anual).

A partir da instalação do experimento até o ano 2008, foram realizadas anualmente no mês de julho medições do diâmetro a altura do peito (DAP) em todas as árvores das parcelas. Os dados foram fornecidos pela empresa BDP.

\section{Modelagem do diâmetro da árvore de área basal média (dg)}

Inicialmente, com os dados de cada parcela e para cada uma das idades avaliadas, foi calculado o diâmetro da árvore de área basal média. A modelagem foi realizada com programa estatístico SAS (Statistical Analysis System) (SAS INSTITUTE, 1999) e teve como forma geral $\mathrm{Y}=$

TABELA 1: Características das faixas de densidade de árvores por hectare avaliadas no experimento de Pinus taeda estabelecido na província de Corrientes, Argentina.

TABLE 1: $\quad$ Characteristics of the blocks with the evaluated tree density (number of trees per hectare) on the trial of Pinus taeda established in Corrientes province, Argentina.

\begin{tabular}{ccccc}
\hline $\begin{array}{c}\text { Faixas } \\
\left(\text { Árvores ha }{ }^{-1}\right)\end{array}$ & $\begin{array}{c}\text { Espaço } \\
\text { disponível } \\
\left(\mathrm{m}^{2} / \text { árvore }\right)\end{array}$ & $\mathrm{N}^{\circ}$ árvores/parcela & $\begin{array}{c}\text { Tamanho da parcela de } \\
\text { medição }\left(\mathrm{m}^{2}\right)\end{array}$ & $\begin{array}{c}\text { Repetições/ } \\
\text { tratamento }\end{array}$ \\
\hline 1666 & 6 & 18 & 108 & 5 \\
833 & 12 & 18 & 216 & 5 \\
416 & 24 & 15 & 360 & 5 \\
208 & 48 & 9 & 432 & 6 \\
\hline
\end{tabular}


$\mathrm{b}_{0}+\mathrm{b}_{1} \mathrm{X}_{1}+\varepsilon$, sendo a variável dependente o diâmetro da árvore de área basal média (dg), a variável independente a idade $(t), b_{0}$ e $b_{1}$ os coeficientes da regressão e $\varepsilon$ o erro estocástico. A homogeneidade de variância foi avaliada pelo teste de aderência de Qui-quadrado. A transformação logarítmica foi utilizada para remover a heterogeneidade da variância é melhorar a precisão da regressão. Foram testados seis modelos (Tabela 2) sendo a variável independente transformada para a forma logarítmica, quadrática e inversa. Os modelos avaliados são usados para ajustar crescimento ou produção em função da idade (SCHNEIDER; SCHNEIDER; MARTINELLI, 2009; BURKHART; TOMÉ, 2012; SCHNEIDER et al., 2014). O modelo foi escolhido em função da precisão estatística, considerando o coeficiente de determinação ajustado ( ${ }^{2}$ aj); o valor de F; o erro padrão $\left(\mathrm{S}_{\mathrm{xy}}\right)$ e o coeficiente de variação expresso em percentagem $(\mathrm{CV} \%)$ para a variável dg.

\section{Análise da covariância (ANACOVA)}

A análise da covariância foi realizada para verificar a existência ou não de diferença significativa de inclinação entre as regressões. Caso não existisse diferença de inclinação, o segundo teste foi realizado para verificar a não diferença de níveis entre as regressões (SCHNEIDER; SCHNEIDER; MARTINELLI, 2009). O teste foi realizado pelo procedimento GLM do SAS. O modelo geral usado para avaliar as inclinações foi:

$\mathrm{Y}=\mathrm{X}+\mathrm{X}^{*} \mathrm{AR}+\mathrm{X}^{*} \mathrm{AR} * \mathrm{NP}+\mathrm{X}^{*} \mathrm{AR} * \mathrm{NP} * \mathrm{CR}$
E o modelo usado para avaliar os interceptos foi: $\mathrm{Y}=\mathrm{X}+\mathrm{AR}+\mathrm{AR} * \mathrm{NP}+\mathrm{AR} * \mathrm{NP} * \mathrm{CR}$

Sendo $\mathrm{Y}$ a variável dependente e $\mathrm{X}$ a covariável. Uma vez obtidas as regressões dos 30 tratamentos avaliados, os resultados do dg em função da idade para as diferentes combinações de ARxNPxCR foram interpretados. Foi considerado adequado reestimar coeficientes quando o comportamento de alguns tratamentos apresentou desvios com relação ao comportamento geral observado. Com o uso dos coeficientes das regressões obtidos, foram geradas funções para estimar os coeficientes da função geral pelo método stepwise. Dessa maneira, obtiveramse 2 modelos que estimam os coeficientes da função geral em função do número de árvores remanescentes após o desbaste pré-comercial (AR), da percentagem de copa remanescente (CR) e do número de podas $(\mathrm{NP})$.

\section{Validação do modelo}

A validação do comportamento do modelo foi realizada com dados independentes daqueles usados na construção do modelo, sendo estes provenientes de parcelas do experimento que não foram consideradas na geração do modelo, e dados de outro experimento, localizado num sitio similar ao do local do presente estudo. Foram considerados os dg $(\mathrm{cm})$ obtidos durante o período de crescimento compreendido entre os 3 e 15 anos.

Os critérios estatísticos usados para validar o modelo de regressão foram apresentados em detalhe por Schneider, Schneider e Martinelli (2009). A metodologia empregada tem sido utilizada

TABELA 2: Modelos de regressão testados para a seleção de uma equação da variável dg em função da variável t.

TABLE 2: Regression models tested for the selection of an equation of the variable $\mathrm{dg}$ in function of $\mathrm{t}$ variable.

\begin{tabular}{cl}
\hline Número & \\
\hline 1 & $\ln (\mathrm{dg})=\mathrm{b}_{0}+\mathrm{b}_{1}(1 / \mathrm{t})$ \\
2 & $\ln (\mathrm{dg})=\mathrm{b}_{0}+\mathrm{b}_{1} \mathrm{t}+\mathrm{b}_{2}(1 / \mathrm{t})$ \\
3 & $\ln (\mathrm{dg})=\mathrm{b}_{0}+\mathrm{b}_{1} \ln (\mathrm{t})+\mathrm{b}_{2} \mathrm{t}^{2}$ \\
4 & $\ln (\mathrm{dg})=\mathrm{b}_{0}+\mathrm{b}_{1}\left(1 / \mathrm{t}^{2}\right)$ \\
5 & $\ln (\mathrm{dg})=\mathrm{b}_{0}+\mathrm{b}_{1}\left(1 / \mathrm{t}^{2}\right)+\mathrm{b}_{2} \mathrm{t}$ \\
6 & $\ln (\mathrm{dg})=\mathrm{b}_{0}+\mathrm{b}_{1} \ln (\mathrm{t})+\mathrm{b}_{2}(\ln (\mathrm{t}))^{2}$ \\
\hline
\end{tabular}

Em que: $\ln =$ logaritmo neperiano; $d g=$ diâmetro da árvore de área basal media; $t=$ idade; $b_{0}, b_{1}$ e $b_{2}$ os coeficientes da regressão. 
por pesquisadores como Vaccaro et al. (2003) e Dominguez, Bravo e Del Rio (2006). Os critérios estatísticos considerados foram: a) Tendência absoluta (BIAS); b) Tendência Relativa (BIAS\%); c) Desvio médio (D); d) Desvio médio relativo (D\%); e) Desvio médio absoluto em percentagem (e\%) e f) Qui-Quadrado $\left(\mathrm{c}^{2}\right)$. As estatísticas mencionadas foram calculadas para o diâmetro da árvore de área basal média (dg). Os dados independentes foram obtidos de parcelas com desbaste pré-comercial para 1296 árvores/ha e 3 desramas anuais do 50\% da copa viva; parcelas com desbaste pré-comercial para 926 árvores/ha e 3 desramas anuais do 50\% da copa viva e parcelas permanentes com 1323 árvores/ ha sem realização de desbastes, nem podas.

\section{RESULTADOS E DISCUSSÃO}

Na Tabela 3 são apresentados os coeficientes e os parâmetros estatísticos de cada equação. O modelo selecionado foi o número 1 , o mesmo apresentou o maior coeficiente de determinação $(0,87)$ e o maior valor do F calculado $(12838,1)$ dos modelos avaliados. Na análise da variância, o baixo número de coeficientes levou à obtenção de um alto valor dos quadrados médios da regressão. O modelo apresentou um elevado valor de $\mathrm{F}$ originado por um alto valor dos quadrados médios da regressão e baixo valor do quadrado médio do erro. Os valores de erro padrão da estimativa e coeficiente de variação em percentagem para a variável $\mathrm{dg}$ foram similares entre os modelos sendo de 4,58 e 19,82, respectivamente para o modelo escolhido.

$\mathrm{Na}$ Tabela 4 é apresentada a análise da covariância da função escolhida, na qual se observa a existência de interação altamente significativa entre a inversa da idade, AR, NP e CR, indicando diferenças significativas nas inclinações das regressões.

A matriz de (b0 e b1) coeficientes das regressões dos 30 tratamentos estudados encontra se apresentada na Tabela 5. Pode-se observar que, na medida em que o número de árvores por hectare diminui, o coeficiente b0 aumenta e o coeficiente b1 diminui.

Os modelos obtidos para estimar os coeficientes $\mathrm{B}_{0}$ e $\mathrm{B}_{1}$ do modelo geral (modelo de regressão aninhado) são os seguintes: 1) $\mathrm{B}_{0}=\mathrm{a}+$ $\mathrm{b} \ln \mathrm{AR}+\mathrm{c} \mathrm{CR}$; 2) $\mathrm{B}_{1}=\mathrm{a}+\mathrm{b} / \mathrm{CR}+\mathrm{c} \mathrm{NP} \mathrm{NP}^{2}+\mathrm{d}$ $\ln \mathrm{AR}$. Os valores dos coeficientes e as estatísticas do modelo constam na Tabela 6 .

Os coeficientes $\mathrm{B}_{0}$ e $\mathrm{B}_{1}$ foram calculados para as combinações correspondentes aos 30 tratamentos avaliados. Os resultados apresentados na Figura 1 mostram claramente como os valores de dg aumentaram dado que o número de árvores

TABELA 3: Modelos de regressão e parâmetros estatísticos testados para descrever o diâmetro da árvore de área basal média (dg) em função da idade ( $\mathrm{t}$ ) para Pinus taeda no nordeste de Corrientes, Argentina.

TABLE 3: Evaluated regression models and statistics parameters to describe the quadratic mean diameter (dg) in function of age ( $\mathrm{t}$ ) for Pinus taeda in the northeast of Corrientes, Argentina.

\begin{tabular}{ccccccccc}
\hline N. & \multicolumn{1}{c}{ Modelo } & $\mathrm{b}_{0}$ & $\mathrm{~b}_{1}$ & $\mathrm{~b}_{2}$ & $\mathrm{R}_{\text {aj. }}^{2}$ & $\mathrm{~S}_{\mathrm{xy}}$ & $\mathrm{CV}(\%)$ & $\mathrm{F}$ \\
\hline 1 & $\mathrm{Lndg}=\mathrm{b}_{0}+\mathrm{b}_{1}(1 / \mathrm{t})$ & 3,8455 & $-5,5879$ & & 0,87 & 4,58 & 19,82 & 12838,1 \\
2 & $\begin{array}{l}\mathrm{Lndg}=\mathrm{b}_{0}+\mathrm{b}_{1} \mathrm{t}+\mathrm{b}_{2} \\
(1 / \mathrm{t})\end{array}$ & 1,2526 & 0,3379 & $-0,0129$ & 0,85 & 4,57 & 19,75 & 5702,73 \\
& $\begin{array}{l}\mathrm{Lndg}=\mathrm{b}_{0}+\mathrm{b}_{1} \mathrm{Ln} \mathrm{t} \\
+\mathrm{b}_{2} \mathrm{t}^{2}\end{array}$ & 0,7087 & 1,2616 & $-0,003$ & 0,87 & 4,56 & 19,68 & 6359,91 \\
4 & $\mathrm{Lndg}=\mathrm{b}_{0}+\mathrm{b}_{1}\left(1 / \mathrm{t}^{2}\right)$ & 3,4216 & $-14,0698$ & & 0,82 & 5,15 & 22,49 & 8874,37 \\
5 & $\begin{array}{l}\mathrm{Lndg}=\mathrm{b}_{0}+\mathrm{b}_{1}\left(1 / \mathrm{t}^{2}\right) \\
+\mathrm{b}_{2} \mathrm{t}\end{array}$ \\
$\begin{array}{l}\mathrm{Lndg}=\mathrm{b}_{0}+\mathrm{b}_{1} \ln \mathrm{t}+ \\
\mathrm{b}_{2} \ln ^{2} \mathrm{t}\end{array}$ & $\begin{array}{c}-0,0674 \\
\mathrm{~ns}\end{array}$ & 2,31032 & $-0,36989$ & 0,87 & 4,57 & 19,76 & 6487,49 \\
\hline
\end{tabular}

Em que: N. = Número do modelo; $\mathrm{Ln} \mathrm{dg}=$ Logaritmo neperiano do diâmetro da árvore de área basal média $(\mathrm{cm}) ; \mathrm{t}=$ Idade (anos); $b_{0}=$ Coeficiente linear; $b_{1}$ e $b_{2}=$ Coeficientes angulares; $\mathrm{R}^{2}{ }_{\text {aj. }}=$ Coeficiente de determinação ajustado; $\mathrm{S}_{\mathrm{xy}}$ : Erro padrão da estimativa; CV $(\%)=$ Coeficiente de variação em percentagem; $\mathrm{F}=$ Valor de $\mathrm{F}$ calculado; $\mathrm{ns}=\mathrm{Não}$ significativo pelo teste de $\mathrm{t}$. 
TABELA 4: Análise da covariância da função $\ln (\mathrm{dg})=\mathrm{B}_{0}+\mathrm{B}_{1} \mathrm{t}^{-1}$, em função do número de árvores remanescentes após desbaste pré-comercial, número de podas e percentagem de copa remanescente para Pinus taeda no nordeste Argentino.

TABLE 4: $\quad$ Covariance analyses of the function $\ln (\mathrm{dg})=\mathrm{B}_{0}+\mathrm{B}_{1} \mathrm{t}^{-1}$, in relation with the number of remnant trees after pre commercial thinning, number of punning and percentage of remnant crown for Pinus taeda in the Argentinean northeast.

\begin{tabular}{lccccc}
\hline \multicolumn{1}{c}{ Fonte Variação } & GL & SQ erro Tipo I & QM & F & Prob. $>\mathrm{F}$ \\
\hline Modelo & 30 & 373,6155 & 12,4538 & 591,11 & $<, 0001$ \\
$\mathrm{t}^{-1}$ & 1 & 359,8433 & 359,8433 & 17079,6 & $<, 0001$ \\
$\mathrm{t}^{-1 *} \mathrm{AR}$ & 3 & 7,3106 & 2,4369 & 115,66 & $<, 0001$ \\
$\mathrm{t}^{-1 *} \mathrm{AR} * \mathrm{NP}$ & 12 & 2,6082 & 0,2173 & 10,32 & $<, 0001$ \\
$\mathrm{t}^{-1 *} \mathrm{AR} * \mathrm{NP} * \mathrm{CR}$ & 14 & 3,8533 & 0,2752 & 13,05 & $<, 0001$ \\
Erro & 1932 & 40,7046 & 0,0211 & & $<, 0001$ \\
Total corrigido & 1962 & 414,3201 & & & \\
$\mathrm{R}^{2}=0,90$ & $\mathrm{CV}(\%)=4,7$ & & & & \\
\hline
\end{tabular}

Em que: $\mathrm{t}^{-1}=$ inverso da idade (anos); $\mathrm{AR}=$ número de árvores remanescentes após o desbaste; $\mathrm{NP}=$ número de podas; $\mathrm{CR}=$ percentagem de copa viva remanescente após a realização da poda; $\mathrm{B}_{0}=$ intercepto; $\mathrm{B}_{1}=$ Coeficiente angular; $\mathrm{GL}=$ Graus de liberdade; $\mathrm{SQ}=$ Soma de quadrados; $\mathrm{QM}=$ Quadrado médio; $\mathrm{F}=$ valor de $\mathrm{F}$ calculado para a variável dependente; Prob. > F = nível de probabilidade do erro; $\mathrm{R}^{2}=$ Coeficiente de determinação.

por ha foi menor após o desbaste pré-comercial (no sentido vertical da Figura 1). A variação do dg encontrada, aos 15 anos, entre os tratamentos de 1666 árvores/ha e 208 árvores/ha sem poda, foi de $15,8 \mathrm{~cm}$. Com relação aos tratamentos de podas, pode ser observado que sendo a copa remanescente foi menor, o dg também foi menor e, porque aumentou o número de podas de frequência anual (no sentido horizontal da Figura 1), os diâmetros foram diminuindo. A redução da capacidade fotossintética das árvores causada pela poda afetou negativamente o crescimento em dg sendo a perda mais significativa na medida em que a desrama foi mais intensa e prolongada. Nas maiores densidades de árvores/ha observaram-se menores diferenças de crescimento em dg entre tratamentos podados e a testemunha sem poda ( $100 \%$ de copa remanescente) devido à poda natural da testemunha nessas condições. Com o acréscimo do peso do desbaste foram obtidas árvores de maiores dimensões, sendo este resultado esperado devido à maior disponibilidade de água, luz e nutrientes por árvore.

As diferenças em dg entre tratamentos sem poda e podados, obtidas aos 15 anos, variaram de $0,74 \mathrm{~cm}$ até $3,90 \mathrm{~cm}$. Os resultados poderiam ser explicados considerando que a remoção de copa viva por meio das podas produz uma redução da capacidade fotossintética da árvore e, consequentemente, isso se traduz em menor crescimento. Quando o número de árvores/ha foi elevado (1666), as árvores não podadas atingiram um comprimento de copa similar ao das árvores podadas a idades mais jovens, sendo este o motivo pelo qual as diferenças observadas em dg foram menores que aquelas observadas nos tratamentos com poucas árvores/ha, nos quais as árvores podadas demoraram mais tempo para atingir um comprimento de copa similar ao das não podadas.

Os resultados obtidos com a remoção de copa viva podem ser comparados com os encontrados por Mowat (1947) em plantios de Pinus ponderosa nos Estados Unidos: após 5 anos da realização da poda, o incremento diamétrico foi diminuindo na medida em que a remoção da copa viva foi aumentando, atingindo diferenças de até $1,9 \mathrm{~cm}$ em diâmetro entre o tratamento com poda de $75 \%$ da copa viva e a testemunha sem poda.

Schneider, Finger e Hoppe (1999), ao avaliarem o efeito da desrama em plantio de Pinus elliottii de 13 anos com densidade inicial de 2000 árvores por hectare e desbastado aos 11 anos, obtiveram, após 4 anos da realização das desramas, diferenças de $1,17 \mathrm{~cm}$ de diâmetro médio entre a testemunha e o tratamento com desrama de $60 \%$ da altura total da árvore. Estes efeitos foram similares aos alcançados no presente estudo, para as podas 
TABELA 5: $\quad$ Matriz de coeficientes (b0 e b1) das regressões dos 30 tratamentos estudados. TABLE 5: $\quad$ Matrix of the regression coefficients (b0 e b1) for the 30 studied treatments.

\begin{tabular}{|c|c|c|c|c|c|}
\hline $\mathrm{T}$ & $\mathrm{AR}$ & NP & $\mathrm{CR}$ & b0 & b1 \\
\hline 1 & 1666 & 1 & 100 & 3,5003 & $-4,1900$ \\
\hline 2 & 1666 & 2 & 70 & 3,4843 & $-4,1900$ \\
\hline 3 & 1666 & 3 & 70 & 3,4812 & $-4,1900$ \\
\hline 4 & 1666 & 4 & 70 & 3,4825 & $-4,1900$ \\
\hline 5 & 1666 & 2 & 50 & 3,4680 & $-4,1900$ \\
\hline 6 & 1666 & 3 & 50 & 3,4617 & $-4,1900$ \\
\hline 7 & 1666 & 4 & 50 & 3,4643 & $-4,1900$ \\
\hline 8 & 833 & 1 & 100 & 3,7394 & $-4,8695$ \\
\hline 9 & 833 & 2 & 70 & 3,7058 & $-4,8695$ \\
\hline 10 & 833 & 3 & 70 & 3,7146 & $-4,8695$ \\
\hline 11 & 833 & 4 & 70 & 3,6802 & $-4,8923$ \\
\hline 12 & 833 & 2 & 50 & 3,6811 & $-4,8695$ \\
\hline 13 & 833 & 3 & 50 & 3,6891 & $-4,8695$ \\
\hline 14 & 833 & 4 & 50 & 3,6173 & $-4,9184$ \\
\hline 15 & 416 & 1 & 100 & 3,9289 & $-5,5173$ \\
\hline 16 & 416 & 2 & 70 & 3,9284 & $-5,7106$ \\
\hline 17 & 416 & 3 & 70 & 3,9276 & $-5,8062$ \\
\hline 18 & 416 & 4 & 70 & 3,9289 & $-6,0973$ \\
\hline 19 & 416 & 2 & 50 & 3,9289 & $-5,9192$ \\
\hline 20 & 416 & 3 & 50 & 3,9289 & $-6,1305$ \\
\hline 21 & 416 & 4 & 50 & 3,9289 & $-6,3944$ \\
\hline 22 & 208 & 1 & 100 & 4,1085 & $-5,9422$ \\
\hline 23 & 208 & 2 & 70 & 4,1078 & $-6,1504$ \\
\hline 24 & 208 & 3 & 70 & 4,1085 & $-6,2652$ \\
\hline 25 & 208 & 4 & 70 & 4,1085 & $-6,5370$ \\
\hline 26 & 208 & 2 & 50 & 4,1085 & $-6,3763$ \\
\hline 27 & 208 & 3 & 50 & 4,1085 & $-6,8347$ \\
\hline 28 & 208 & 4 & 50 & 4,1085 & $-7,2178$ \\
\hline 29 & 208 & 2 & 30 & 4,1085 & $-6,8061$ \\
\hline 30 & 208 & 3 & 30 & 4,1085 & $-7,5804$ \\
\hline
\end{tabular}

Em que: $\mathrm{T}=$ Tratamento; $\mathrm{AR}=$ número de árvores remanescentes após o desbaste; $\mathrm{NP}=$ número de podas; $\mathrm{CR}=$ percentagem de copa viva remanescente após a realização da poda; $b 0=$ intercepto; $b 1=$ coeficiente angular.

realizadas na densidade de 1666 plantas/ha.

Resultados semelhantes aos obtidos para os tratamentos de poda e desbaste foram apresentados por Keller, Fassola e Pachas (2004), ao avaliarem tratamentos de podas com 3 intervenções de frequência anual e remoção da copa viva em percentagens de 30,50 e $70 \%$, em um experimento de Pinus taeda, de 11 anos de idade, localizado na província de Misiones, Argentina. Os resultados indicaram que o aumento de remoção de copa viva diminuiu o crescimento. Quando a poda foi de $70 \%$, as diferenças observadas foram da ordem de 2 até $5 \mathrm{~cm}$ do DAP, aumentando as diferenças quando a densidade de árvores por hectare foi menor. Os desbastes estudados foram realizados aos 3 anos de idade, partindo de uma densidade de 1960 plantas por hectare, remanescendo 980, 490 e 245 árvores/ ha. Variações próximas a 4, 10 e $16 \mathrm{~cm}$ foram encontradas nos tratamentos sem poda quando comparados com a testemunha (1960 árvores/ha). 
TABELA 6: Coeficientes e parâmetros estatísticos obtidos para estimar os coeficientes $\mathrm{B}_{0}$ e $\mathrm{B}_{1}$ do modelo geral para avaliar o dg $(\mathrm{cm})$ para povoamentos de Pinus taeda no nordeste de Corrientes, Argentina.

TABLE 6: Coefficients and statistics parameters obtained to estimate $\mathrm{B}_{0}$ and $\mathrm{B}_{1}$ coefficients of the general model for $\mathrm{dg}(\mathrm{cm})$ estimation in Pinus taeda stands in the northeast of Corrientes, Argentina.

\begin{tabular}{cccccccc}
\hline N. & $\mathrm{a}$ & $\mathrm{b}$ & $\mathrm{c}$ & $\mathrm{d}$ & $\mathrm{R}^{2}$ & $\mathrm{CV}(\%)$ & $\mathrm{F}$ \\
\hline 1 & 5,7312 & $-0,3080$ & 0,0005 & ------ & 0,99 & 0,54 & 2039,3 \\
2 & $-12,0219$ & $-33,3441$ & $-0,0188$ & 1,1510 & 0,95 & 4,34 & 169,5 \\
\hline
\end{tabular}

Em que: N. = Número do modelo; lnAR $=$ logaritmo neperiano do número de árvores remanescentes/ha; $\mathrm{CR}=$ percentagem de copa viva remanescente após a realização da poda; $\mathrm{NP}=$ número de podas; $\mathrm{a}=$ Coeficiente linear; $b$, c, $d=$ Coeficientes angulares; $\mathrm{R}^{2}=$ Coeficiente de determinação; CV (\%) = Coeficiente de variação em percentagem; $\mathrm{F}=$ Valor de $\mathrm{F}$ calculado.

Masatoshi e Velez Mesa (1992), estudando o efeito da poda realizada aos 3,5 anos de idade em plantios de Pinus patula na Colômbia, encontraram, após 4 anos, que o tratamento com $70 \%$ de remoção da copa viva apresentou um volume inferior a $40 \%$ com relação ao tratamento de $30 \%$ de remoção de copa viva. Segundo os autores, o volume estava diretamente relacionado ao dg, sendo constatado que as árvores podadas mostraram, da mesma maneira que no presente estudo, menores dg que as não podadas.

$\mathrm{O}$ efeito de diferentes intensidades do desbaste pré-comercial no crescimento em dg ao longo do tempo foi simulado e representado na Figura 2, na qual pode ser observado como, na medida em que as densidades de árvores/ha foram maiores, os dg foram menores, gerando uma diferença diamétrica cada vez maior ao longo do tempo entre densidades.

A informação gerada a partir dos modelos é de utilidade para conhecer o dg no tempo para uma densidade escolhida e definir o momento do próximo desbaste usando valores de área basal crítica ou diagramas de manejo de densidade próprios da espécie como ferramentas.

As diferenças crescentes observadas na Figura 2 podem ser explicadas pelos diferentes níveis de concorrência existentes entre as árvores, sendo maior nas densidades maiores. Os valores do dg alcançados, aos 15 anos de idade, variaram de $25,9 \mathrm{~cm}$ (1600 árvores/ha) até 41,8 cm (200 árvores/ ha). A diferença obtida entre esses valores foi $61,4 \%$ sobre o valor de dg de 1600 árvores/ha.

Resultados similares aos encontrados neste estudo foram obtidos por Crechi et al. (2005) para Pinus taeda, aos 15 anos de idade, na província de Misiones, Argentina, que, ao avaliarem diversos tratamentos de desbastes, obtiveram que a testemunha sem desbaste e densidade inicial de 1736 árvores/ha apresentou um diâmetro médio de $24,4 \mathrm{~cm}$. Já os tratamentos com desbastes realizados a cada 2 anos, removendo 33 e $66 \%$ da área basal, apresentaram diâmetros de $30,3 \mathrm{~cm}$ e de $46,1 \mathrm{~cm}$, respectivamente. Os tratamentos com desbastes contavam com 462 e 100 árvores/ha aos 15 anos de idade. Também foi apresentado que o tratamento com desbaste de $66 \%$ da área basal e desbastes realizados a cada 6 anos apresentou 189 árvores/ha aos 15 anos de idade e um diâmetro médio de 39,3 $\mathrm{cm}$, sendo as respostas do Pinus taeda a diferentes graus de competição muito similares no presente estudo.

Comparando-se os resultados obtidos com a citação realizada no parágrafo anterior foi possível observar que, embora as diferenças máximas atingidas em $\mathrm{dg}(\mathrm{cm})$ tenham sido elevadas, seria possível atingir maiores valores se o número de árvores/ha fosse inferior a 208 plantas, consequentemente, pode-se inferir que as 208 plantas/ha estavam competindo por recursos, tais como luz, água e nutrientes.

O estudo realizado por Sayer et al. (2004) em um plantio de Pinus taeda de 17 anos no estado de Louisiana, nos Estados Unidos, permitiu observar que os tratamentos sem desbaste $(2990$ árvores/ha) apresentaram um DAP de $15 \mathrm{~cm}$, enquanto o tratamento com desbastes realizados aos 7 (749 árvores remanescentes/ha) e 14 anos (31\% do máximo valor de Índice de densidade de Reineke para Pinus taeda) apresentou um DAP médio de $23 \mathrm{~cm}$. Os desbastes foram efetivos em mover a distribuição diamétrica para as maiores 
classes, gerando produtos para serraria, enquanto, no tratamento sem desbastes, os produtos obtidos foram para a polpa. Ao serem comparados esses resultados com os do presente estudo, pode-se inferir que, além das diferenças nas respostas aos desbastes, os diferentes locais e tempos de execução

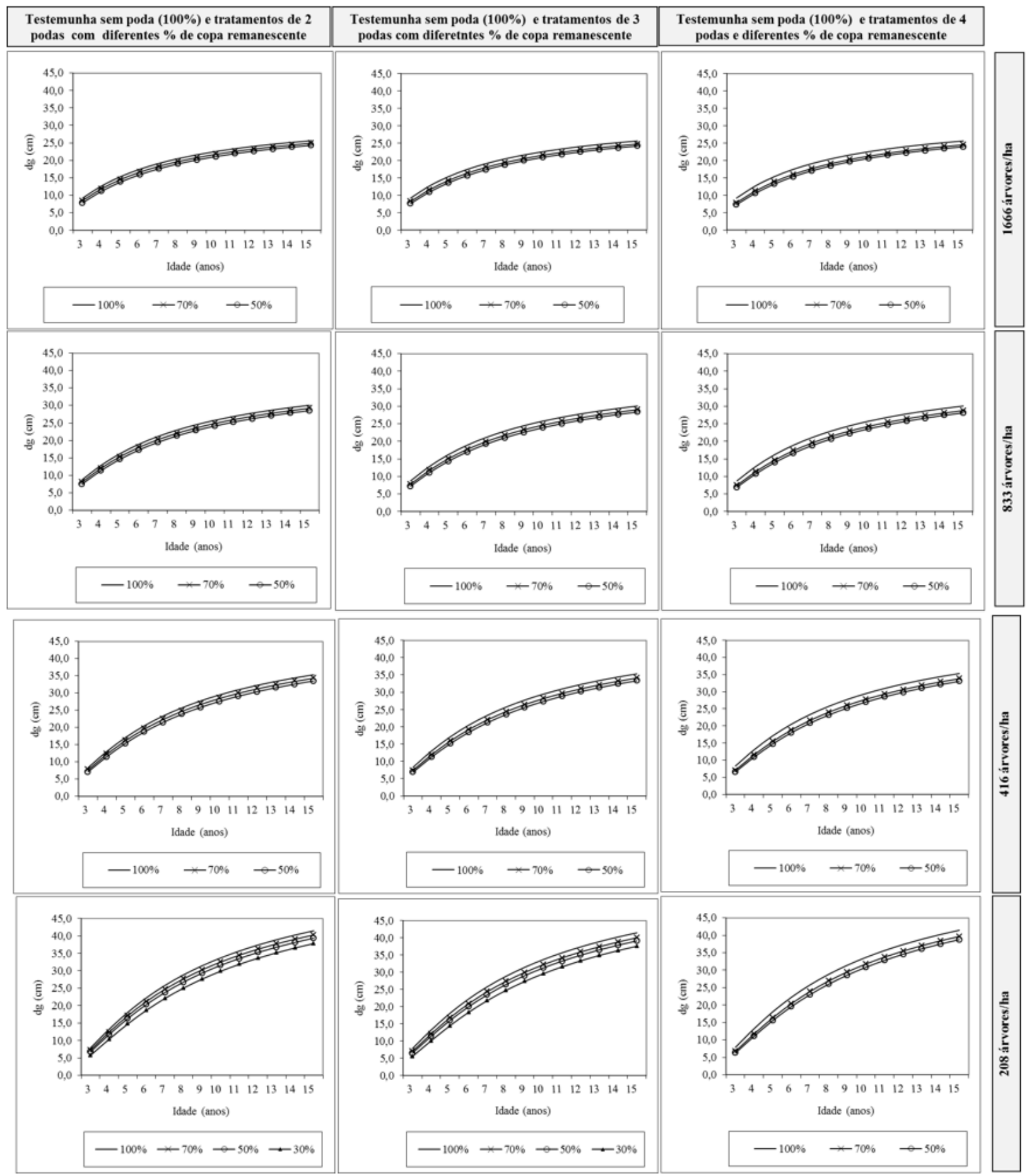

FIGURA 1: Crescimento diamétrico em função da idade para 4 densidades de árvores/ha com a testemunha sem poda e podas de frequência anual por dois, três e quatro anos e diferentes \% de copa remanescente para Pinus taeda no nordeste de Corrientes, Argentina.

FIGURE 1: Diametric growth in function of age for 4 densities of trees/ha with a check without pruning and annual pruning for two, three and four years with different \% of remnant crown for Pinus taeda in the northeast of Corrientes, Argentina. 


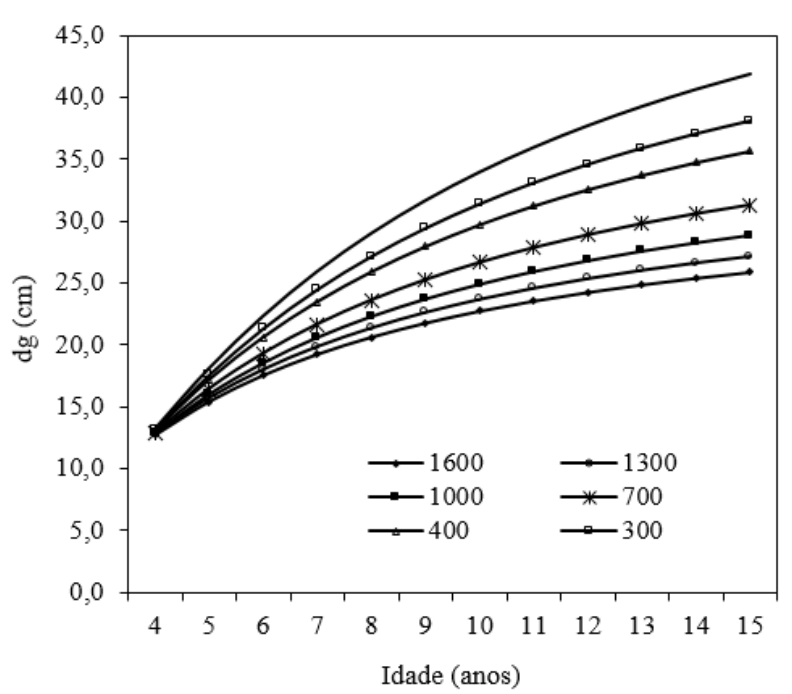

FIGURA 2: $\quad$ Simulação do crescimento em dg (cm) em função da idade (anos) para diferentes densidades de árvores/ha originadas pela realização de um desbaste pré-comercial, aos 3 anos de idade, sem podas, para Pinus taeda no nordeste de Corrientes, Argentina.

FIGURE 2: $\quad$ Dg $(\mathrm{cm})$ growth simulation in function of age (years) for different densities (trees/ha) originated by pre-commercial thinning at 3 year old, without pruning for Pinus taeda in the northeast of Corrientes, Argentina.

dos desbastes influem nas respostas de crescimento em diâmetro.

Tendências similares foram apresentadas por Leite, Gilciano e Moreira (2006), ao avaliarem o efeito do espaçamento no crescimento de Pinus taeda até os 14 anos de idade, no estado de Santa Catarina. Os autores verificaram que, quando a densidade foi de 1333 árvores/ha, o dg alcançou um valor de $24,1 \mathrm{~cm}$ sendo este valor inferior em $10,4 \%$ com relação ao dg simulado no presente estudo para 14 anos e 1300 árvores/ha. Os pesquisadores observaram, como neste trabalho que a estagnação do crescimento em diâmetro iniciou mais cedo nos menores espaçamentos, uma vez que esse crescimento foi substancialmente afetado pela densidade. As maiores taxas de crescimento foram obtidas nos espaçamentos mais amplos.

Também, as respostas de Pinus taeda a diferentes espaçamentos foram estudadas por Harms,
Whitesell e Debell (2000) no Hawaii, onde foram observadas tendências similares às apresentadas neste estudo para o crescimento em dg. Aos 20 anos de idade, os diâmetros médios obtidos foram de $24,9 \mathrm{~cm}$ para uma densidade inicial de 1615 árvores por hectare e de $32,7 \mathrm{~cm}$ para 746 árvores/ha.

Os pesquisadores Sanquetta et al. (2003), estudando o crescimento e a produção de Pinus taeda com 12 anos de idade sob diferentes espaçamentos iniciais de plantio, concluíram que os maiores espaçamentos conferem um aumento acentuado no DAP. Para as densidades de 1428 e 909 árvores/ha, os valores médios de DAP foram de 20,7 e $24,0 \mathrm{~cm}$, respectivamente. Essa variação foi semelhante, mas inferior à encontrada no presente estudo entre as densidades de 1666 e 833 árvores/ ha sendo provável que a causa disto seja a maior variação de árvores/ha.

Os modelos apresentados poderão ser usados pelos produtores para avaliar diferentes manejos, conforme os objetivos de produção (celulose, madeira para serraria, laminação) e estimar a rentabilidade de diferentes regimes para a seleção do mais conveniente. Ao se conhecer o efeito do desbaste e das podas no crescimento em diâmetro será possível saber qual é a rotação necessária para lograr o diâmetro objetivo com diversos regimes de manejo, por exemplo, um plantio de Pinus taeda com 833 árvores/ha de Pinus taeda e desbaste précomercial para 416 árvores/ha com podas de $50 \%$ de remoção de copa viva durante 3 anos permitirá obter produtos para serraria e laminação em rotações de 15 anos de idade com diâmetros médios superiores a $30 \mathrm{~cm}$.

\section{Validação do modelo}

Os resultados do teste de validação presentes na Tabela 7 indicam que os valores de $\mathrm{c}^{2}$ foram todos não significativos, indicando não haver diferença estatística significativa com $99 \%$ de confiabilidade na distribuição dos valores observados na amostra de validação e dos valores estimados pelo modelo.

Os valores obtidos de BIAS; BIAS\%; D; $\mathrm{D} \%$ e e $\%$, apresentados na Tabela 7 , indicaram que os modelos podem ser usados devido a sua elevada precisão, sendo o maior valor de desvio médio absoluto em percentagem de 5,71.

Da análise do $\mathrm{D} \%$, pode-se observar que, nos cenários 1 e 3, o modelo subestimou, enquanto no caso do cenário 2 , o modelo superestimou. 
TABELA 7: Resultados dos testes de validação do modelo geral (lndg $\left.=\mathrm{B}_{0}+\mathrm{B}_{1} \mathrm{t}^{-1}\right)$ para estimação do $\mathrm{dg}(\mathrm{cm})$ em três cenários de manejo para plantios de Pinus taeda no nordeste de Corrientes, Argentina.

TABLE 7: $\quad$ Results of the validation tests of the general model $\left(\operatorname{lndg}=\mathrm{B}_{0}+\mathrm{B}_{1} \mathrm{t}^{-1}\right)$ for $\mathrm{dg}(\mathrm{cm})$ estimation in 3 management situations for Pinus taeda plantations in the northeast of Corrientes, Argentina.

\begin{tabular}{cccccccc}
\hline Cenário & Descrição & $\begin{array}{c}\text { Tendência } \\
\text { absoluta } \\
\text { (BIAS) }\end{array}$ & $\begin{array}{c}\text { Tendência } \\
\text { relativa } \\
(\text { BIAS\%) }\end{array}$ & $\begin{array}{c}\text { Desvio } \\
\text { médio } \\
(\mathrm{D})\end{array}$ & $\begin{array}{c}\text { Desvio } \\
\text { médio } \\
\text { relativo } \\
(\mathrm{D} \%)\end{array}$ & $\begin{array}{c}\text { Desvio médio } \\
\text { absoluto em } \\
\text { percentagem } \\
(\mathrm{e} \%)\end{array}$ & $\begin{array}{c}\text { Qui- } \\
\text { quadrado } \\
\left(\mathrm{c}^{2}\right)\end{array}$ \\
\hline 1 & $1296-\mathrm{CR} 30-2 \mathrm{NP}$ & $-0,47$ & $-2,58$ & $-0,03$ & $-3,23$ & 2,86 & $0,44 \mathrm{~ns}$ \\
2 & $926-\mathrm{CR} 30-2 \mathrm{NP}$ & 0,94 & 4,86 & 0,04 & 4,14 & 5,65 & $0,95 \mathrm{~ns}$ \\
3 & 1323 -sem poda & $-0,74$ & $-3,85$ & $-0,04$ & $-3,54$ & 5,71 & $0,78 \mathrm{~ns}$ \\
\hline
\end{tabular}

Em que: $\mathrm{B}_{0}=5,7312-0,3080 \ln \mathrm{AR}+0,00053 \mathrm{CR} ; \mathrm{B}_{1}=-12,0219-33,3441 / \mathrm{CR}-0,0188 \mathrm{NP}^{2}+1,151 \operatorname{lnAR}$; $\mathrm{dg}=$ diâmetro da árvore de área basal média $(\mathrm{cm}) ; \mathrm{t}$ = idade (anos); $\mathrm{ns}=$ não significativo.

\section{CONCLUSÕES}

a) Os desbastes pré-comerciais afetam positivamente o crescimento do diâmetro da árvore de área basal média, enquanto as podas o afetam de maneira negativa.

b) $\mathrm{O}$ crescimento em dg diminui com o aumento da percentagem de remoção de copa viva e do número de podas.

c) As diferenças de crescimento em diâmetro médio geradas pelo desbaste são crescentes no tempo e aumentam como o peso do desbaste.

d) As podas têm uma menor influência no crescimento em dg quando as densidades de árvores/ ha são elevadas (1666 árvores/ha).

e) Os modelos obtidos na presente pesquisa apresentaram boa precisão (desvio médio absoluto em percentagem igual a 5.71) podendo ser usados para estimar o dg de plantios de Pinus taeda no nordeste de Argentina.

f) O produtor florestal poderá usar os modelos obtidos para estimar o tempo necessário para atingir um diâmetro médio objetivo e usar essa informação para avaliar a rentabilidade de diferentes cenários de manejo. Também, os modelos poderão ser usados para estimar o dg no tempo para uma densidade de árvores por hectare e determinar o momento oportuno para desbastar o povoamento usando diagramas de manejo de densidade (DMD) da espécie.

\section{AGRADECIMENTOS}

Os autores agradecem a Forestal
Bosques del Plata S.A. e ao Instituto Nacional de Tecnologia Agropecuária pela concessão de dados dendrométricos e ao Ing. A. Hernández pela colaboração na instalação do experimento, condução e medição das árvores. Os autores agradecem à Capes e ao CNPq pela colaboração na realização do presente trabalho.

\section{REFERÊNCIAS BIBLIOGRÁFICAS}

AMATEIS, R. L.; BURKHART, H. The influence of thinning on the proportion of peeler, sawtimber, and pulpwood tress in Loblolly pine plantations. South African Journal of Applied Forestry, Durban, v. 29, n 3, p. 158-162. 2005.

BURKHART, H. E.; TOMÉ, M. Modeling Forest Trees and Stands. New York: Springer, 2012. 457 p.

UNIVERSDAD NACIONAL DE ENTRE RÍOS. Facultad de Ciencias Agropecuarias. Cátedra de Climatologia Agricola. Clasificaciones climáticas. 2010. Disponível em: $<$ http://www.cicyttp.org. ar/climatologiafca/docencia/apuntes/tema8.pdf $>$. Acesso em: 2 abr. 2010.

COSTAS, R. et al. Modelos de la producción de Pinus taeda empleando variables vinculadas con las podas. Bosque, Valdivia, v. 27, n. 2, p. 98-107, 2006.

CRECHI, E. H. et al. Efectos del raleo en Pinus taeda. Idia XXI, Buenos Aires, v. 5, n. 8, p. 43-47, 2005.

CURTIS, R. O.; MARSHALL, D. D. Why Quadratic Mean Diameter? Western Journal of Applied Forestry, Maryland, p. 137-139, 2000. 
DOMÍNGUEZ, M. D.; BRAVO, F.; DEL RIO, M. Modelos de copa de Pinus sylvestris L. em bosques del centro de España. Interciencia, Caracas, v. 31, n. 3, p. 168-175, 2006.

ELIZONDO, M. Primer inventario forestal de la

Provincia de Corrientes. Provincia de Corrientes: Consejo Federal de Inversiones, 2009. 69 p. ESCOBAR, J. et al. Mapa de suelos de la provincia de Corrientes. Corrientes: Gobierno de la provincia de Corrientes; Instituto Nacional de Tecnología Agropecuaria (INTA), 1996. $429 \mathrm{p}$.

FASSOLA, H. et al. Evolución de las principales variables de árboles de Pinus taeda L. sometidos a diferentes tratamientos silviculturales en el nordeste de la provincia de Corrientes, Argentina. Ciência Florestal, Santa Maria, v. 12, n. 2, p. 51-60, 2002. FINGER, C. A. Fundamentos de biometría florestal. Santa Maria: [s. n.], 1992. 269 p.

HARMS, W.; WHITESELL, C.; DEBELL, D. Growth and development of loblolly pine in a spacing trial planted in Hawaii. Forest Ecology and Management, Amsterdam, v. 126, p. 13-24, 2000.

KELLER, A.; E.; FASSOLA, H. E.; PACHAS, N. Efecto de la poda y raleo sobre el crecimiento de Pinus taeda L. a los 11 años de edad en el departamento de Iguazú, Misiones. In: JORNADAS TÉCNICAS FORESTALES Y AMBIENTALES, 11., Eldorado, Anais... Eldorado: FCF, UNAM, EEA Montecarlo INTA, 2004. 8 p.

LARSON, P. R. et al. Formation and properties of juvenile wood in southern pines. A synopsis. [s. 1.]: USDA Forest Service, 2001. 42 p. (General Technical report, 129).

LEITE, H. G.; GILCIANO, S. N.; MOREIRA, A. Efeito do espaçamento e da idade sobre variáveis de povoamentos de Pinus taeda L. Arvore, Viçosa, MG, v. 30, n. 4, p. 603-612, 2006.

MASATOSHI, E.; VELEZ MESA, G. Results of a pruning trial with Pinus patula in Colômbia. IPEF International, Piracicaba, v. 2, p. 45-49, 1992.

MOWAT, E. Effect of pruning on growth of Ponderosa pine. Forest research notes, Oregon, v. 38, n. 3, 1947.

SANQUETTA, C. et al. Produção de madeira livre de nós em povoamentos de Pinus taeda em função da densidade de plantio. Cerne, Lavras, v. 9, n. 2, p. 129-140, 2003.

SAS INSTITUTE. SAS/STAT User's guide. Version 8 (computer manual). Cary: SAS Institute, 1999.

SAYER, M. A. S. et al. Long-term trends in Loblolly pine productivity and stand characteristics in response to thinning and fertilization in the West Gulf region. Forest ecology and management, Amsterdam, v. 192, p. 71-96, 2004.

SCHNEIDER, P. R. et al. Crescimento em diâmetro do Pinus elliottii e Pinus taeda em áreas arenizadas e degradadas no Oeste do Rio Grande do Sul. Ciência Rural, Santa Maria, v. 44, n. 9, p. 1561-1567, 2014. SCHNEIDER, P. R.; FINGER, C. A.; HOPPE, J. M. Efeito da intensidade de desrama na produção de Pinus elliottii Engelm implantado em solo pobre, no estado do Rio Grande do Sul. Ciência Florestal, Santa Maria, v. 9, n. 1, p. 35-46, 1999.

SCHNEIDER, P. R. ; SCHNEIDER, P. S. P. Introdução ao manejo florestal. Santa Maria: FACOS-UFSM, 2008. 566 p.

SCHNEIDER, P. R. ; SCHNEIDER, P. S. P. ; MARTINELLI, C. A. S. Análise de regressão aplicada à Engenharia Florestal. 2. ed. Santa Maria: FACOS-UFSM, 2009. 294 p.

TASSISA, G.; BURKHART, H. Modeling thinning effects on rings width distribution in loblolly pine (Pinus taeda). Canadian Journal of Forest Research, Canada, n. 27, p. 1291-1301, 1997.

UNITED STATES DEPARTMENT OF AGRICULTURE. Soil Taxonomy - a basic system of soil classification for making and interpreting soil surveys. 2. ed. Washington, DC: USDA, 1999. 870 p.

VACCARO, S. et al. Incremento em área basal de árvores de uma floresta estacional decidual, em três fases sucessionais, no município de Santa Tereza, RS. Ciência Florestal, Santa Maria, v. 13, n. 2, p. 131-142, 2003. 